\title{
Paraptosis triggers mitochondrial pathway-mediated apoptosis in Alzheimer's disease
}

\author{
DONG-PEI JIA*, SONG WANG* ${ }^{*}$, BAO-CHAO ZHANG and FANG FANG \\ Department of Neurological Rehabilitation, Nanyang City Center Hospital, Nanyang, Henan 473003, P.R. China
}

Received March 8, 2015; Accepted May 8, 2015

DOI: 10.3892/etm.2015.2531

\begin{abstract}
In previous years, increasing evidence has indicated that paraptosis and mitochondrial-mediated apoptosis may be associated with Alzheimer's disease (AD). However, the association between paraptosis and mitochondrial-mediated apoptosis, and the pathological processes underlying AD, remain elusive. In the present study, the $\beta$-amyloid precursor protein gene, and the gene mutations PS1M146L and L286V, were transfected to an SH-SY5Y cell line to establish an AD cell model. Subsequently, an MTT assay was used to examine the cell viability of the AD cell model, while a TUNEL assay was employed to observe the number of positively stained apoptotic cells. Cytoplasmic vacuolization was examined using light microscopy and images were photographed. Furthermore, western blot analysis was utilized to detect the expression of golden biomarkers of the mitochondrial pathway, including Bcl-2 and Bax. The paraptosis inhibitor, cycloheximide, was selected to treat the AD model cells in order to observe the association between paraptosis and mitochondrial-mediated apoptosis. The results indicated that the decrease in the cell viability of the AD cells was initiated at $24 \mathrm{~h}$, as compared with the normal cells $(\mathrm{P}<0.05)$. TUNEL-positive stained cells were observed at $48 \mathrm{~h}$, which was later compared with the cell death initiation. In addition, examination of eytoplasmic vacuolization using microscopy indicated that there were a small number of paraptosis cells present at $24 \mathrm{~h}$. The expression levels of Bcl-2 was significantly decreased, while Bax was significantly increased at $48 \mathrm{~h}$. Furthermore, cycloheximide treatment was demonstrated to significantly increase $\mathrm{Bcl}-2$ expression, while decreasing Bax expression ( $\mathrm{P}>0.05)$. In conclusion, the occurrence of paraptosis was demonstrated in the early pathological
\end{abstract}

Correspondence to: Dr Bao-Chao Zhang, Department of Neurological Rehabilitation, Nanyang City Center Hospital, 312 Gongnong Road, Nanyang, Henan 473003, P.R. China

E-mail: zhangbaocny@yeah.net

"Contributed equally

Key words: Alzheimer's disease, paraptosis, mitochondria-mediated apoptosis, cycloheximide, Bcl-2 stages of AD, which may subsequently damage the mitochondria and trigger mitochondrial pathway-mediated apoptosis. Thus, paraptosis may trigger programmed cell death directly, or indirectly through the regulation of $\mathrm{Bcl}-2$ and Bax protein expression.

\section{Introduction}

Alzheimer's disease (AD), known to be the leading cause of dementia in elderly populations in clinical practice, is a neurodegenerative disease that is characterized by a progressive loss of memory and cognitive function (1). The main characteristic of AD is the formation of extracellular senile plaques, which include $\beta$-amyloid precursor protein (APP) and intracellular neurofibrillary tangles $(2,3)$. The pathological mechanism underlying AD has been investigated for a number of years; however, the essential cause of the disease has not been fully elucidated. In previous years, increasing evidence has indicated that the mitochondrial pathway may trigger the apoptosis of cells in AD patients (1-3).

Previous studies have been indicated that AD could induce the formation of vacuoles (4), and the formed vacuoles may be associated with cell death. Cell death primarily comprises two main types, including programmed cell death (PCD) and passive (necrotic) cell death (5). Under physiological conditions, apoptosis and autophagy are the two main types of PCD. Furthermore, non-lysosomal vacuolated degeneration (also known as paraptosis) is a novel type of PCD, which is characterized by cytoplasmic vacuolization derived from endoplasmic reticulum and mitochondria swelling; however, there is a shortage of apoptotic morphology (4).

In a preliminary study, brain cells were not found to be apoptotic prior to mitochondrial-triggered apoptosis under TUNEL analysis. Therefore, it was hypothesized that there were a number of necessary processes prior to cells undergoing mitochondrial pathway-mediated apoptosis. Thus, the aim of the present study was to investigate the association between mitochondrial pathway meditated-apoptosis and paraptosis.

\section{Materials and methods}

Establishment of an AD cell model. The APP gene of (Swedish, Florida, London) strain and PS1M146L and L286V mutations were transfected into a SH-SY5Y cell line (American Type Culture Collection, Manassas, VA, USA). The transfec- 
tion of the above genes and mutations was performed using Lipofectamine 2000 transfection reagent (Invitrogen Life Technologies, Carlsbad, CA, USA). According to the identification of amyloid- $\beta$, the results demonstrated that the AD cell model had been established successfully.

All animal experiments were performed in accordance with the guidelines of the Laboratory Animal Ethical Standards of Nanyang City Center Hospital (Nanyang, China).

MTT assay. AD cell models were seeded and cultured in 96-well plates at a density of $2 \times 10^{4}$ cells $/ \mathrm{ml}$ in complete medium and incubated overnight. The cell viability was detected using an MTT assay (Sigma-Aldrich, St. Louis, MO, USA), as described previously (6). The MTT assay was performed at different time points, which included 24, 48 and $72 \mathrm{~h}$.

Sample treatment and western blot analysis. AD model cells were homogenized gently in an isolation buffer containing $0.25 \mathrm{M}$ sucrose, $10 \mathrm{mM}$ HEPES-NaOH, (pH 7.4) and $1 \mathrm{mM}$ EDTA. Subsequently, the samples were homogenized in a prechilled lysis buffer $(150 \mathrm{mM} \mathrm{NaCl}, 50 \mathrm{mM}$ Tris- $\mathrm{HCl}$, $1 \%$ Nonidet P-40, $0.25 \%$ sodium deoxycholate, $0.1 \%$ SDS, $1 \mathrm{mM}$ PMSF, $10 \mathrm{mg} / \mathrm{ml}$ leupeptin, $1 \mathrm{mM} \mathrm{Na} \mathrm{VO}_{4}$ and $1 \mathrm{mM}$ $\mathrm{NaF}$ ) overnight at $4{ }^{\circ} \mathrm{C}$. The homogenates were collected and centrifuged at $12,000 \mathrm{x} \mathrm{g}$ for $20 \mathrm{~min}$. The brain samples were subjected to $15 \%$ SDS-PAGE, and the obtained proteins in the gels were transferred to polyvinylidene difluoride membranes (Millipore Corporation, Temecula, CA, USA). The membranes were incubated with the following primary antibodies, mouse anti-human Bax monoclonal (1:3,000; Santa Cruz Biotechnology, Inc., Dallas, TX, USA), mouse anti-human Bcl-2 monoclonal (1:4,000; Santa Cruz Biotechnology, Inc.) and mouse anti- $\beta$-actin $(1: 4,000$; Santa Cruz Biotechnology, Inc.) at $4^{\circ} \mathrm{C}$ overnight. Subsequently, the membranes were washed and incubated with a horseradish peroxidase-conjugated secondary rabbit anti-mouse antibody (1:3,000; Santa Cruz Biotechnology, Inc.) for $2 \mathrm{~h}$ at room temperature. Immunoreactive bands were visualized with the SuperSignal West Pico Chemiluminescent Substrate (Pierce Biotechnology, Inc., Rockford, IL, USA) using ChemDoc XRS with Quantity One software (Bio-Rad Laboratories, Inc., Hercules, CA, USA).

Detection of cytoplasmic vacuolization. AD cells were cultured on 24-well plates. At the time points of 24, 48 and $72 \mathrm{~h}$, the cells were visualized using a CX31 light microscope (Olympus Corporation, Tokyo, Japan) and images were photographed. AD and normal cells were analyzed using a washout assay and phase contrast microscopy, according to the protocol described in a previous study (4). Light and fluorescent microscopic images were selected and recorded from the representative fields of the cells plates at the different time points. All the experiments were performed a minimum of three times independently, and all the images were photographed in the same manner.

Transmission electron microscopy analysis of cytoplasmic vacuolization. The structure of the brain cells was observed utilizing a transmission electron microscope (H-600IV;
Hitachi, Ltd., Tokyo, Japan), as reported in a previous study (7).

Statistical analysis. Every experiment was repeated a minimum of three times. The average value of the repeated data was expressed as the mean \pm standard error of the mean. Statistical comparisons were performed using the Student's t-test with SPSS 19.0 software (SPSS IBM, Armonk, NY, USA), where $\mathrm{P}<0.05$ was considered to indicate a statistically significant difference.

\section{Results}

Cell viability of the $A D$ cell model and normal cells. In order to investigate the effects of double transgenesis on the viability of the AD cell model, the cell viability was examined using an MTT assay. The MTT results indicated that a decrease in the cell viability of the AD cells was observed initially at $24 \mathrm{~h}$ (Fig. 1A). However, the cell viability of the AD cells at 48 and $72 \mathrm{~h}$ was significantly decreased when compared with the normal cells (Fig. 1A; $\mathrm{P}<0.05$ ).

Apoptosis observations in the AD cell model. A TUNEL assay was performed to detect the rate of apoptosis in the AD cells and normal cells. The results indicated that TUNEL-positive stained cells were observed at $48 \mathrm{~h}$, which was later compared with the occurrence of cell death (Fig. 1B). In addition, the number of TUNEL-positive cells at $72 \mathrm{~h}$ was significantly increased when compared with the number at $24 \mathrm{~h}$ (Fig. 1B; $\mathrm{P}<0.05)$. However, no TUNEL-positive cells were observed in the normal cell samples at any of the time points.

Paraptosis observations during the early growth stages of the AD cells. In order to investigate the specific pathological mechanism underlying the apoptosis of AD cells, the AD cells were visualized using light microscopy and images were photographed. The results revealed that there were a small number of paraptosis cells observed under microscopy at the 24-h time point (Fig. 2). Furthermore, the number of paraptosis cells was shown to increase with an increase in culture time, with significantly increased numbers observed at 48 or $72 \mathrm{~h}$ when compared with the number at $24 \mathrm{~h}$ (Fig. 2). However, there were no paraptosis cells observed in any of the normal cell samples cultured at the different time points.

Changes in the expression of Bcl-2 and Bax during the late growth stage of $A D$ cells. The role of mitochondrial pathway-mediated apoptosis in AD was also investigated by analyzing the protein expression levels of Bcl-2 and Bax using western blot analysis. The results indicated that the expression levels of Bcl-2 at $48 \mathrm{~h}($ Fig. 3A; $\mathrm{P}<0.05)$ and $72 \mathrm{~h}$ (Fig. 3; $\mathrm{P}<0.01)$ were significantly decreased when compared with the level at $24 \mathrm{~h}$. With regard to Bax protein expression, the levels were significantly increased at $48 \mathrm{~h}$ (Fig. $3 \mathrm{~A} ; \mathrm{P}<0.01)$ and $72 \mathrm{~h}$ (Fig. 3; $\mathrm{P}<0.01$ ) when compared with the expression level at $24 \mathrm{~h}$.

Paraptosis inhibitor blocks mitochondrial pathway-mediated apoptosis. In order to confirm the induction of mitochondrial pathway-mediated apoptosis with paraptosis as the 
A

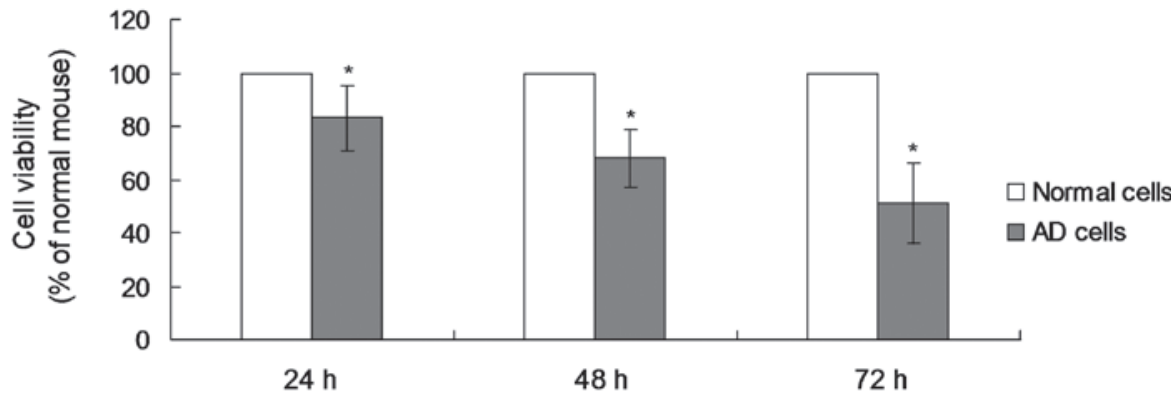

B

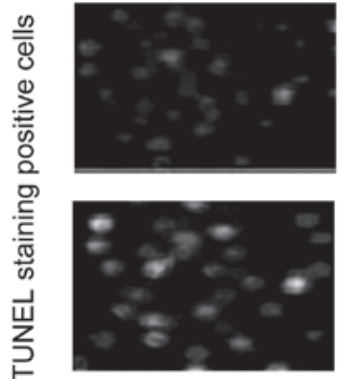

$24 \mathrm{~h}$

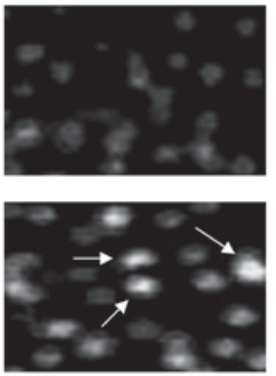

$48 \mathrm{~h}$

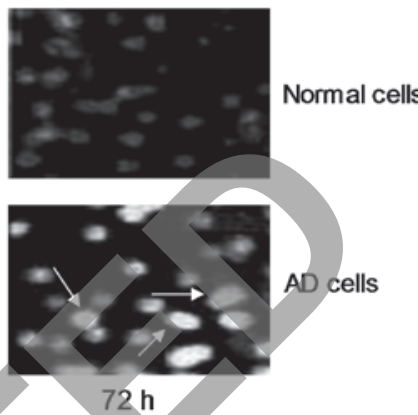

Figure 1. Observations of cell viability and apoptosis in the AD model cells. (A) Cell viability detection using the MTT assay. (B) A TUNEL assay was used to assess the number of apoptosis-positive cells. "P<0.05, vs. normal cells. The arrows in Fig. $1 B$ indicate the TUNEL-positive stained cells. AD, Alzheimer's disease.

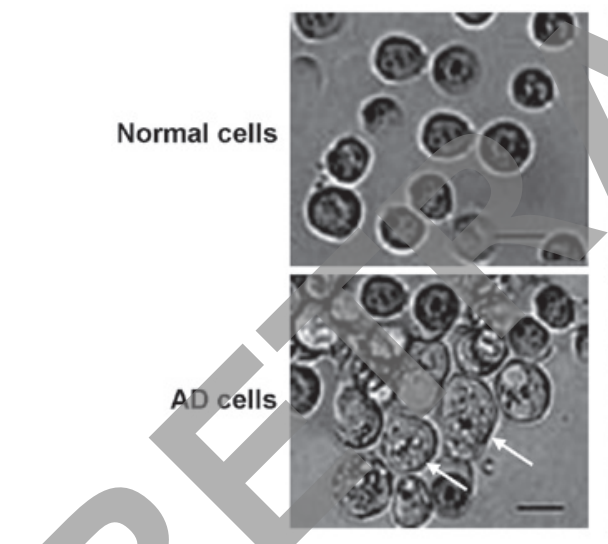

$24 \mathrm{~h}$

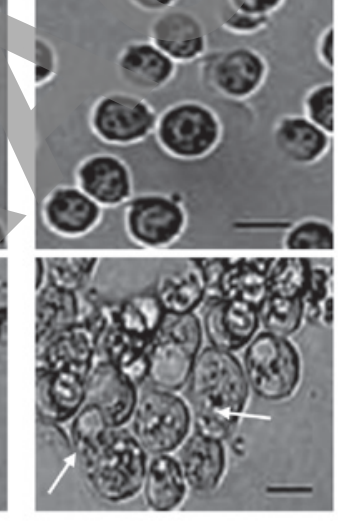

$48 \mathrm{~h}$

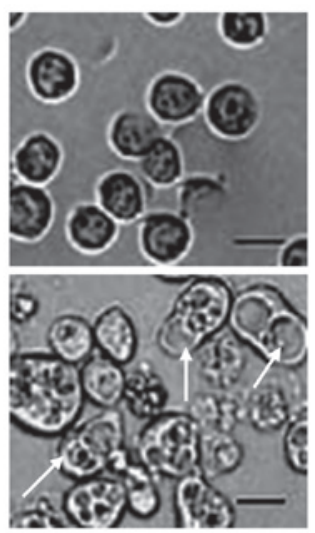

$72 \mathrm{~h}$

Figure 2. Cytoplasmic vacuolization was observed in the AD cells. AD and normal cells were analyzed by a washout assay using phase contrast microscopy at the indicated time points. The arrows indicate the cells exhibiting cytoplasmic vacuolization (magnification, $\mathrm{x} 400$; scale bar, $30 \mu \mathrm{m}$ ). AD, Alzheimer's disease.

trigger, an inhibitor of paraptosis, namely cycloheximide (Sigma-Aldrich), was added to the cell cultures. The results revealed that cycloheximide treatment significantly increased the expression levels of Bcl-2, while decreasing Bax expression at 48 or $72 \mathrm{~h}$ when compared with the expression levels at 24 h (Fig. 3B; P>0.05).

\section{Discussion}

Previous studies have reported a number of mechanisms for the cell death and apoptosis of AD cells (2-4). However, the specific processes of apoptosis in AD have not been fully elucidated. Therefore, the present study investigated the details of the apoptotic pathway in AD pathology.

Paraptosis is a recently defined as a form of PCD (7); however, the underlying mechanism has not been fully investi- gated. To the best of our knowledge, there have been no previous studies investigating the interactions between paraptosis and other types of PCD in the pathogenesis of AD disease. Thus, the aim of the present study was to investigate the association between paraptosis and mitochondrial pathway-mediated apoptosis in the pathogenic processes underlying AD.

Previous studies have indicated that extensive cytoplasmic vacuolization may be associated with necrosis and necroapoptosis $(8,9)$. Damage to the cell membrane is one of the characteristics of paraptosis (9). Paraptosis is defined as a form of cell death, with the characteristics of cytoplasmic vacuolization, cellular swelling, membrane blebbing and increased membrane permeability $(10,11)$. Wang et al (12) demonstrated that paraptosis triggers retinal ganglion cell death via the production of reactive oxygen species, and hypothesized that paraptosis may be associated with mitochondrial damage. 
A

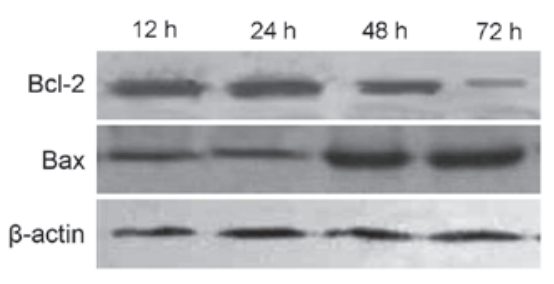

B

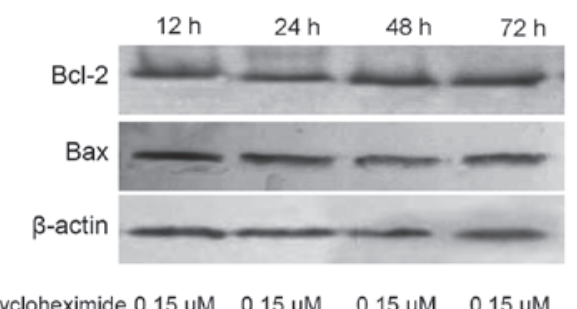

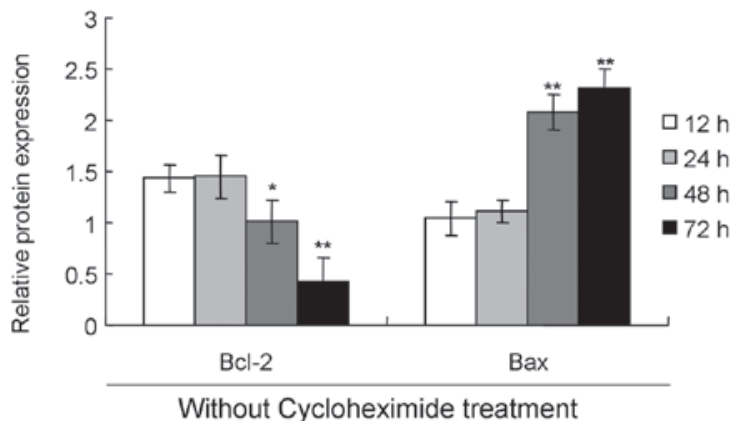

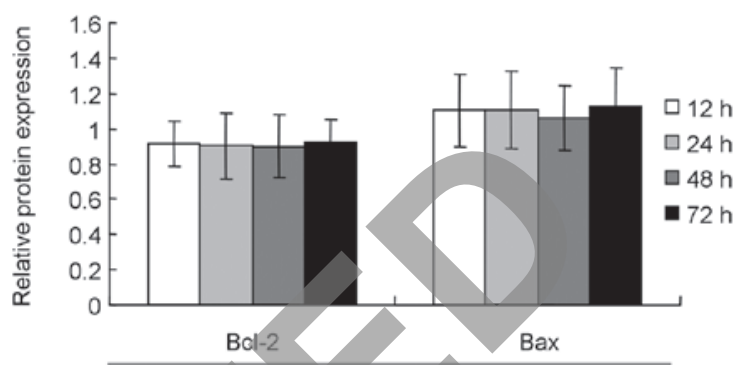

Figure 3. Western blot analysis showing the protein expression of Bcl-2 and Bax with and without cycloheximide treatment. (A) Protein expression levels of Bcl-2 and Bax following treatment with cycloheximide. (B) Protein expression levels of Bcl-2 and Bax without cycloheximide treatment. "P $<0.05$ and ${ }^{* *} \mathrm{P}<0.01$, vs. $24 \mathrm{~h}$.

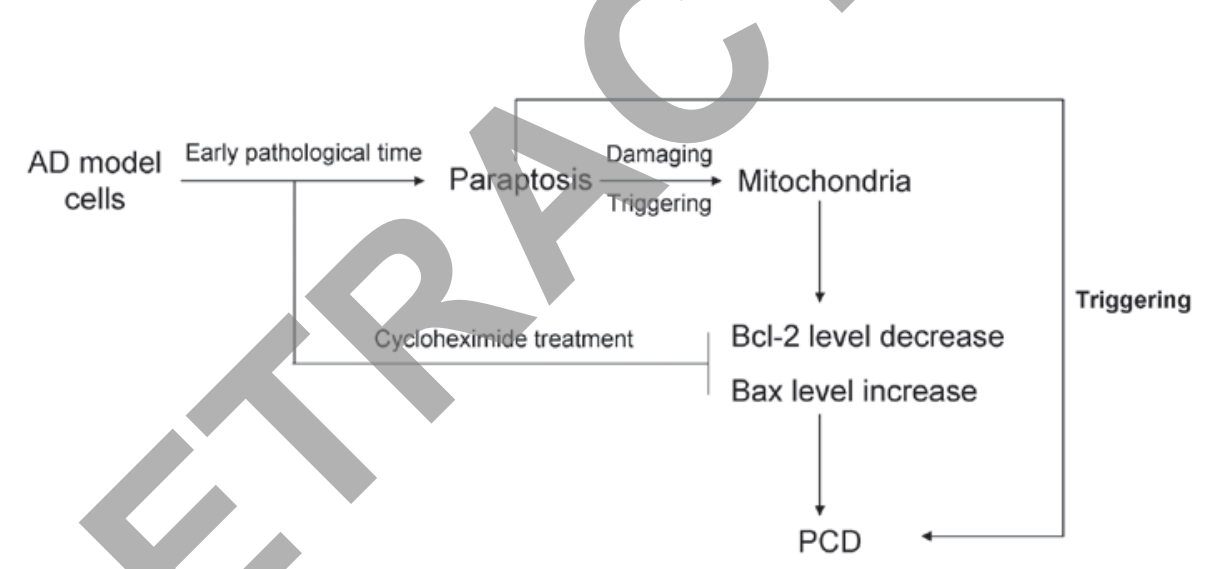

Figure 4. Diagram showing the hypothesis for paraptosis-triggered mitochondrial pathway-mediated apoptosis in AD cells. AD, Alzheimer's disease; PCD, programmed cell death.

Bcl-2 and Bax proteins are members of the Bcl family of proteins $(13,14)$. A number of studies have reported that the Bcl-2 family proteins participate in $\mathrm{AD}$, and are associated with cell apoptosis $(15,16)$. In particular, Bcl-2 protein is known to inhibit a variety of apoptotic pathways, and in the majority of cases, Bcl-2 is considered to function through the inhibition of Bax protein (17). Therefore, the association between paraptosis and mitochondrial pathway-mediated apoptosis was investigated with the aim to further elucidate the pathogenic processes underlying $\mathrm{AD}$.

In the present study, the cell viability of the AD cell model was found to significantly decrease when compared with the normal cells. However, the decrease in cell viability was initiated after culture for $24 \mathrm{~h}$. In order to investigate the reasons underlying the decrease in cell viability for the AD cells, the extent of apoptosis was detected using a TUNEL assay. However, the TUNEL assay results indicated that apoptosis was initiated until the 48 -h time point. Thus, the MTT and
TUNEL results demonstrated that the apoptosis of the cells may occur prior to necrosis. Therefore, in the following experiments, the extent of paraptosis and mitochondrial pathway mediated-apoptosis was investigated.

The results of the present study indicated that paraptosis was initiated after $24 \mathrm{~h}$ of culture in the AD model cells. However, mitochondrial pathway-mediated apoptosis was initiated after $48 \mathrm{~h}$ of cell culture. Therefore, the PCD that occurs during the early stages of AD (24 h) was hypothesized to not be the result of mitochondrial mediated-apoptosis, but the result of paraptosis. In addition, the results indicated that paraptosis may trigger mitochondrial pathway-mediated apoptosis. In order to confirm this hypothesis, an inhibitor of paraptosis, namely cycloheximide (18), was applied as treatment to the AD cells. Following treatment of the $\mathrm{AD}$ cells with cycloheximide, the expression of $\mathrm{Bcl}-2$ was shown to increase, while the protein expression of Bax protein was found to decrease, with results similar to those observed in the normal cells. In future studies, 
cycloheximide may be applied in animals to investigate its effects on the rate of paraptosis in vivo.

Therefore, according to the aforementioned results, a hypothesis was established with regard to the pathogenic process underlying $\mathrm{AD}$. In the early pathological stages of $\mathrm{AD}$, paraptosis occurs, which may damage the mitochondria with disease progression. Subsequently, the damaged mitochondria induce a decrease in $\mathrm{Bcl}-2$ expression and an increase in Bax expression, which are key biomarkers for mitochondrial pathway-mediated apoptosis. Furthermore, the paraptosis inhibitor (8), cycloheximide, was demonstrated to block paraptosis, and consequently inhibit the changes in Bcl-2 and Bax protein expression (Fig. 4).

In conclusion, paraptosis was demonstrated to occur during the early pathological stages of $\mathrm{AD}$, which subsequently damaged the mitochondria and triggered mitochondrial pathway-mediated apoptosis. Therefore, paraptosis was shown to trigger PCD directly, or indirectly through the regulation of Bcl-2 and Bax protein expression.

\section{References}

1. Zhu Y, Li C, Sun A, Wang Y and Zhou S: Quantification of microRNA-210 in the cerebrospinal fluid and serum: Implications for Alzheimer's disease. Exp Ther Med 9: 1013-1017, 2015.

2. Chambers JK, Uchida K, Harada T, Tsuboi M, Sato M, Kubo M, Kawaguchi $\mathrm{H}$, Miyoshi N, Tsujimoto $\mathrm{H}$ and Nakayama $\mathrm{H}$ : Neurofibrillary tangles and the deposition of a beta amyloid peptide with a novel-N-terminal epitope in the brains of wild Tsushima leopard cats. PLoS One 7: e46452, 2012.

3. Guo J, Chang L, Zhang X, Pei S, Yu M and Gao J: Ginsenoside compound $\mathrm{K}$ promotes $\beta$-amyloid peptide clearance in primary astrocytes via autophagy enhancement. Exp Ther Med 1271-1274, 2014.

4. Yu WH, Kumar A, Peterhoff C, Shapiro Kulnane L, Uchiyama Y, Lamb BT, Cuervo AM and Nixon RA: Autophagic vacuoles are enriched in amyloid precursor protein-secretase activities: Implications for beta-amyloid peptide over-production and localization in Alzheimer's disease. Int J Biochem Cell Biol 36: 2531-2540, 2004.

5. Sosna J, Voigt S, Mathieu S, Lange A, Thon L, Davania P, Herdegen T, Linkermann A, Rittger A, Chan FK, Kabelitz D, et al: TNF-induced necroptosis and PARP-1 mediated necrosis represent distinct routes to programmed necrotic cell death. Cell Mol Life Sci 71: 331-348, 2014
6. Karl R, Singha PK, Venkatachalam MA and Saikumar P: A novel role for MAPI LC3 in nonautophagic cytoplasmic vacuolation death of cancer cells. Oncogene 28: 2556-2568, 2009.

7. Wang Y, Yang Z and Zhao X: Honokiol induces paraptosis and apoptosis and exhibits schedule-dependent synergy in combination with imatinib in human leukemia cells. Toxico Mech Methods 20: 234-241, 2010.

8. Degterev A, Huang Z, Boyce M, Li Y, Jagtap P, Mizushima N, Cuny GD, Mitchison TJ, Moskowitz MA and Yuan J: Chemical inhibitor of nonapoptotic cell death with therapeutic potential for ischemic brain injury. Nat Chem Biol 1: 112-119, 2005.

9. Kroemer G, El-Deiry WS, Golstein P, Peter ME, Vaux D, Vandenabeele P, Zhivotovsky B, Blagosklonny MV, Malorni W, Knight RA, Piacentini M, et al: Glassification of cell death: Recommendations of the nomenclature committee on cell death. Cell Death Differ 12: 1463-1467, 2005.

10. Majno G and Joris I: Apoptosis, oncosis and necrosis: An overview of cell death. Am J Pathol 146: 3-15, 1995.

11. Trump BF, Berezesky IK, Chang SH and Phelps PC: The pathways of cell death: Oncosis, apoptosis and necrosis. Toxicol Pathol 25: 82-88, 1997

12. Wang Y, Xu K, Zhang H, Zhao J, Zhu X, Wang Y and Wu R: Retinal ganglion cell death is triggered by paraptosis via reactive oxygen species production: A brief literature review presenting a novel hypothesis in glaucoma pathology. Mol Med Rep 10: $1179-1183,2014$.

13. Wang J, Xie Y, Feng Y, Zhang L, Huang X, Shen X and Luo X: (-)-Epigallocatechingallate induces apoptosis in B lymphoma cells via caspase-dependent pathway and $\mathrm{Bcl}-2$ family protein modulation. Int J Oncol 46: 1507-1515, 2015.

14. Ma L and Li W: Emodin inhibits LOVO colorectal cancer cell proliferation via the regulation of the Bcl-2/Bax ratio and cytochrome c. Exp Ther Med 8: 1225-1228, 2014.

15. Yan Y, Gong K, Ma T, Zhang L, Zhao N, Zhang X, Tang P and Gong Y: Protective effect of edaravone against Alzheimer's disease - relevant insults in neuroblastoma N2a. Neurosci Lett 531: 160-165, 2012.

16. Kim JH: Brain-derived neurotrophic factor exerts neuroprotective actions against amyloid beta-induced apoptosis in neuroblastoma cells. Exp Ther Med 8: 1891-1895, 2014.

17. Oakes SA, Scorrano L, Opferman JT, Bassik MC, Nishino M, Pozzan T and Korsmeyer SJ: Proapoptotic Bax and BAK regulate the type 1 inositol trisphosphate receptor and calcium leak from the endoplasmic reticulum. Proc Natl Acad Sci USA 102: 105-110, 2005.

18. Kim SH, Shin HY, Kim YS, Kang JG, Kim CS, Ihm SH, Choi MG, Yoo HJ and Lee SJ: Tunicamycin induces paraptosis potentiated by inhibition of BRAFV600E in FRO anaplastic thyroid carcinoma cells. Anticancer Res 34: 4857-4868, 2014. 